\title{
Editor's Perspective: Basics of an Article Submission
}

Making a difference is the main purpose of health sciences journals nowadays, especially for an open access journal. Open access provides means of publishing rapidly and cost effectively. It has revolutionized substantially in searching latest data in limited time span while providing tools for extracting scientific data and literature to make it more graspable. As the society is expanding and developing, hence the information regarding different issues are now easier to obtain from the latest resources online. Mostly, scientific authors or medical researchers are remarkably skilled in their specified fields, but when it comes to write an article or manuscript, they find it difficult and have limited guidelines of writing scientifically for a journal. Researchers are less aware regarding real cost of publication for example some journals or publishers take submission fee, peer reviewing fee, processing fee and publication fee after or before acceptance in a journal. The author only retains copyright while they can reach largest possible readers. But for the scientist this could be the stepping stone to diversifying or specializing in the field of professionalism to pursue for a career as well.

Irrespective of how incomparable a piece of research article is, if it does not fit into proper criteria of a journal, it may not even be considered for acceptance, peer review or for publication in a journal. Therefore, as an author of a manuscript, one must check the online available articles of that journal before submitting the manuscript. An author must make sure that the contents of the write up best fit the journal or similar to the contents of the journal.

If an author is submitting the manuscript in a multi- disciplinary journal, his or her article can easily be accessed to a vast audience i.e. internationally. Therefore, one must make sure that the manuscript is written in such a customized way that it may be great interest to a large number of audiences. Editors mostly look at the manuscript from the readers point of view and judge accordingly that whether reader finds the manuscript interesting or not and is it useful?

Editor's perspective, therefore is, that the article should be written in a proper scientific language which is related to the field in which the research is conducted. This is followed by the journal's requirement like originality of research work, plagiarism clearance, length of the article, typographical errors, grammar, figures and tables, references and there proper citation within the article, over all formatting along with cover letter according to the submission format. Proper files are then checked by the editor to see whether the author has submitted the material in the word file or PDF. Thereafter, the submitted research work is submitted or forwarded to the external reviewer after the initial desk review is complete. Articles that are found irrelevant are sent back to the authors for further corrections and resubmission if the above criteria are not fulfilled.

These are the things that an editor looks for in an article when it is submitted and are important. These skills can be improved, refined and cultivated by adopting good writing practices among researchers. Introductory courses for manuscript writing must be developed and established in the institutions along with research work and protocol writing.

doi.org/10.21089/njhs.21.0001

Editor

Amna Yahya

NJHS

16, Maqbool Society, Block 7-8,

Lal Muhammad Ch. Road, Karachi - 75350

Web:www.njhsciences.com 